\title{
Autotransplantation de germes dentaires au centre hospitalier de Pau : une série de cas
}

\author{
C. Alande (Bouliac), C. Landric (Pau)
}

Autotransplantation de germes dentaires au centre hospitalier de Pau : une série de cas Alande C1, Landric C2 1. Interne en Chirurgie Orale, UFR Odontologie, Service ORL et Stomatologie CH Pau 2. Spécialiste en Chirurgie Orale, Assistante hospitalière, CH Pau. INTRODUCTION : L'autotransplantation correspond au déplacement d'un organe fonctionnel (transplant) d'un site donneur vers un site receveur, sur un même patient. Dans le cadre de l'organe dentaire, le transplant est placé dans une alvéole osseuse intrabuccale naturelle ou préparée chirurgicalement. Les indications sont nombreuses : délabrement carieux, expulsion traumatique, défaut d'éruption, agénésie. C'est une technique chirurgicale peu utilisée, pourtant les métaanalyses les plus récentes font état d'un taux de succès compris entre 75 et $91 \%$ (1). Ce travail expose une série de 07 transplantations. OBSERVATION : Les 7 transplantations ont été réalisées au Centre Hospitalier de Pau entre aout 2017 et janvier 2018. Les patients étaient initialement adressés par leur dentiste ou leur othodontiste pour des avulsions. Les indications résultaient toutes d'un délabrement carieux de premières molaires maxillaires ou mandibulaires, ces dernières étant non restaurables. Les patients étaient âgés de 17 à 23 ans. Les transplants étaient tous des germes de 3ème molaire incluse situées au stade 7-8 de Nolla. Le même protocole chirurgical a été systématiquement utilisée pour chacun des patients, à savoir : avulsion de la dent délabrée, révision et rinçage alvéolaire, préparation du site receveur, avulsion du germe, temps extra-alvéolaire le plus court possible, positionnement dans le site receveur avec ajustement si nécessaire, mise en sous occlusion par améloplastie, contention. Un soin tout particulier était accordé à la préservation des cellules desmodontales du transplant. Les patients n'ont pas présenté de complication per ou postopératoire. Leur suivi post-opératoire est en cours et est réalisé de façon systématique à 1 semaine, 1 mois, 2 mois avec orthopantomogramme et 6 mois. Pour être considérées comme un succès, les transplantations devaient présenter les critères suivants : poursuite de l'édification radiculaire, absence de mobilité du transplant, absence de signes infectieux cliniques et radiologiques, visualisation radiologique d'un ligament alvéolo-dentaire sans signe d'ankylose. DISCUSSION : De plus en plus d'études tendent à montrer que la préservation des cellules desmodontales est un des facteurs majeurs pour la réussite du traitement (2). Avec l'avènement de la planification 3D (3), ce paramètre pourra être d'avantage contrôlé. Les taux de succès de cette thérapeutique, déjà élevés, pourraient être amenés à augmenter d'avantage. Les transplantations sont aujourd'hui une alternative de choix au traitement implantaire chez les jeunes patients. 1) Machado L A et al. Int journal of oral and max fac surg 2016 May;45(5):610-7 2) Garcia A. Int. Orthodont., 2005, 3 : 221-234 3) Strbac Georg D. Journal of endodont 2016 Dec;42(12):1844-1850 